\title{
OPCIONES REALES EN LA EVALUACIÓN Y MANEJO DE RIESGO EN PROYECTOS DE INVERSIÓN PÚBLICA: CASO MÉXICO
}

\author{
REAL OPTIONS IN THE EVALUATION AND RISK MANAGEMENT IN PUBLIC \\ INVESTMENT PROJECTS: CASE MEXICO \\ Elio Martínez MirandA* \\ Velda Liliana Rodríguez HernándeZ** \\ Universidad Nacional Autónoma de México - UNAM / México, D.F. \\ [Recepción: Setiembre de 2014/ Conformidad: Octubre 2014]
}

\section{RESUMEN}

Desde el enfoque de la evaluación social de proyectos, este artículo expone la forma de cómo se podría usar la metodología de opciones reales en proyectos de inversión pública, y propone la opción social de diferir un proyecto aplicado a un caso de ampliación de un libramiento carretero en Tepic, Nayarit - México.

\section{Palabras clave:}

Opciones reales; evaluación de proyectos; inversión pública.

\begin{abstract}
From the social viewpoint the evaluation of projects in this article presents the way how you could use the methodology of real choices in public investment projects, and proposes the social option of deferring a project applied to a case of extension of a highway bypass in Tepic, Nayarit - Mexico.
\end{abstract}

\section{Keywords:}

Real options; project evaluation; public investment.

* Doctor en Economía. E-mail: elio_mmiranda@hotmail.com

** Maestra en Ingeniería con especialidad en Optimación Financiera. E-mail. inglilianardz@yahoo.com.mx 


\section{INTRODUCCIÓN}

La evaluación social de proyectos es una herramienta de la administración pública, integrada por elementos complementarios de diversas ciencias y técnicas, que se utiliza para apoyar a las autoridades de los países, Estados o municipios en su proceso de toma de decisiones respecto a la asignación de los recursos públicos. Esta herramienta es útil sobre todo cuando se habla de proyectos de inversión pública, e incluso cuando se hace referencia a algunos proyectos privados, que por su magnitud o porque buscan el apoyo o el aval del gobierno, pueden afectar el bienestar o la riqueza de la sociedad en su conjunto.

En lo que respecta a los proyectos de inversión privados, nadie dudaría de la conveniencia de aplicar los criterios de la evaluación privada o financiera.

La evaluación social de proyectos puede hacerse con base en dos tipos de análisis (metodologías): costo-beneficio y costo-eficiencia.

Para evaluar proyectos de infraestructura vial es común que se utilice la metodología costo-beneficio, debido a que ambos (costos y beneficios) son factibles de cuantificar en términos monetarios.

\section{LA TEORÍA DE OPCIONES REALES EN PROYECTOS DE INVERSIÓN PÚBLICA}

La metodología de opciones reales se puede utilizar en la evaluación de un proyecto de inversión pública y podría considerarse como una herramienta de la evaluación social de proyectos.

La incertidumbre y la flexibilidad no se encuentran exclusivamente en los proyectos de los inversionistas privados, sino que también, están presentes en los proyectos del sector público. De esta manera, si en un proyecto del sector público existe incertidumbre y esta disminuye con el tiempo, entonces la metodología de opciones reales se puede aplicar en el análisis de riesgo y en la evaluación de este proyecto.

\section{EVALUACIÓN SOCIAL DE LA AMPLIACIÓN DE UN LIBRAMIENTO CARRETERO EN LA CIUDAD DE TEPIC, NAYARIT, MÉXICO}

En 1980, se terminó de construir en la Ciudad de Tepic, Edo. de Nayarit, un libramiento carretero de aproximadamente $12 \mathrm{~km}$ de longitud. Este libra- miento constó de dos carriles sin acotamientos y tuvo como función principal evitar el tránsito que utilizaba la ruta México-Nogales circulara a través de la zona urbana. Debido al crecimiento de la ciudad y por ende, de la mancha urbana, el libramiento fue alcanzado por esta, lo que ocasionó que los vehículos urbanos utilizaran esta vía como un periférico y que se incrementara los Costos Generalizados de Viaje (CGV) de los vehículos que utilizaban el libramiento. Dada esta problemática, se elaboraron cuatro propuestas de ampliación y mejoramiento del libramiento, siendo la ampliación de dos a cuatro carriles la asignada al Curso Intensivo en Preparación y Evaluación Socioeconómica de Proyectos que realizó el Centro de Estudios para la Preparación y Evaluación Socioeconómica de Proyectos (CEPEP) de México en la Ciudad de Tepic en marzo de 1997.

El proyecto fue propuesto por la Secretaria de Comunicaciones y Transportes (SCT) de México en coordinación con el Gobierno del Estado de Nayarit y la Presidencia Municipal de Tepic (la evaluación social de este proyecto la realizó el CEPEP). La ampliación consistió en construir un nuevo cuerpo adicional de la misma longitud que el actual, con lo cual se contaría con cuatro carriles distribuidos dos en sentido oriente y dos en sentido poniente, separados por un camellón central de 23 metros.

De acuerdo a los estudios que se realizaron, alrededor de 70 calles o avenidas de la ciudad se intersectaban con el libramiento, lo que ocasionaba que el aforo vehicular o el Tránsito Diario Promedio Anual (TDPA) fuera distinto a lo largo de esta vialidad.

De esta manera, considerando lo anterior y las diferentes características geométricas y físicas de la carretera, se establecieron 5 tramos (1A, 1B, 2, 3 y 4) del libramiento obteniéndose el TDPA en cada uno de ellos.

Para poder obtener una aproximación del cálculo de la Tasa de Rentabilidad Inmediata (TRI), el equipo de evaluación prorrateó la inversión total (social) para cada uno de los tramos como se muestra a continuación: 
Cuadro No 01: Monto de Inversión Social para cada Tramo

\begin{tabular}{|c|c|}
\hline Tramo & Monto Social \\
\hline $1 \mathrm{~A}$ & $18,117,493$ \\
\hline $1 \mathrm{~B}$ & $34,685,924$ \\
\hline 2 & $17,165,271$ \\
\hline 3 & $15,992,495$ \\
\hline 4 & $25,227,143$ \\
\hline Total & $\mathbf{1 1 1 , 1 8 8 , 3 2 6}$ \\
\hline
\end{tabular}

Considerando las cifras del Cuadro No 01 y los beneficios netos anuales de cada tramo, en el Cuadro No 02 se muestra una estimación del momento óptimo de inversión social, en donde se supuso que los cinco tramos del libramiento tendrían un período de ejecución de un año.

Fuente: Elaboración propia.

Cuadro No 02: Momento Socialmente Óptimo de Inversión

\begin{tabular}{|c|c|c|c|c|c|}
\hline Tramo & $\begin{array}{c}\text { Beneficio } \\
\text { Neto (\$) }\end{array}$ & $\begin{array}{c}\text { Anulidad de la } \\
\text { Inversión (Ir) }\end{array}$ & $\begin{array}{c}\text { Año Óptimo } \\
\text { de Operación }\end{array}$ & $\begin{array}{c}\text { Año Óptimo } \\
\text { de Inversión }\end{array}$ & $\begin{array}{c}\text { Tasa de Des- } \\
\text { cuento Social }\end{array}$ \\
\hline 1A & $3,300,951$ & $3,261,149$ & 2000 & 1999 & $18 \%$ \\
\hline 1B & $5,567,450$ & $5,549,748$ & 2002 & 2001 & $16 \%$ \\
\hline 2 & $4,052,174$ & $3,089,749$ & 1998 & 1997 & $18 \%$ \\
\hline 3 & $5,004,958$ & $2,878,649$ & 1998 & 1997 & $18 \%$ \\
\hline 4 & $4,425,965$ & $4,036,343$ & 2001 & 2000 & $16 \%$ \\
\hline
\end{tabular}

Fuente: Elaboración propia.

La evaluación social del proyecto del libramiento carretero de la Ciudad de Tepic que se hizo en 1997, determinó que era inmediata la construcción de dos tramos y que para los tres tramos restantes habría que esperar para iniciar su construcción. Por lo tanto, en términos del momento óptimo para invertir y del VPN Social, la evaluación social concluyó el programa de inversiones que se muestra en el siguiente cuadro:

Cuadro No 03: VPN Social de cada tramo del Libramiento

\begin{tabular}{|c|c|c|}
\hline Tramo & $\begin{array}{c}\text { Beneficio } \\
\text { Neto } \mathbf{( \$ )}\end{array}$ & Decisión en base a la TRI \\
\hline 1A & $3,438,774$ & Diferir la inversión 2 años \\
\hline 1B & $-344,206$ & Diferir la inversión 4 años \\
\hline 2 & $10,966,863$ & Invertir ya \\
\hline 3 & $18,754,325$ & Invertir ya \\
\hline 4 & $2,892,574$ & Diferir la inversión 3 años \\
\hline
\end{tabular}

Fuente: Elaboración propia.

De acuerdo al Cuadro No 03, para los tramos 1A, 1B y 4 existía la posibilidad de diferir o posponer la inversión social. Además, con excepción del tramo 1B, el VPN Social estático (pasivo) de los 4 tramos restantes resultó positivo.
La evaluación social de proyectos considera que si se tiene un VPN Social de inicio negativo para los proyectos de vialidades; es decir, que si originalmente no son socialmente rentables, conviene para la entidad ejecutora su postergación durante un cierto período 
de años, con la finalidad de incorporar información real de tal suerte que el VPN Social sea finalmente positivo. Es así, que resulta atrayente considerar la metodología de opciones reales en la evaluación social de proyectos.

En proyectos viales y en base a la evaluación social, el criterio adecuado para saber en qué momento invertir es la Tasa de Rentabilidad Inmediata (TRI), la cual garantiza y determina el año o período en donde se obtiene el "máximo" VPN Social.

El verdadero valor de un proyecto de inversión (público, social y privado) que es analizado desde la perspectiva de la evaluación social, estaría dado por el Valor Presente Neto Extendido Social, en el cual se incluiría el valor de las opciones reales sociales.

Es claro que en la evaluación social de un proyecto de infraestructura vial no se ha considerado el valor de la opción social de diferir. La importancia de dicha opción radica cuando existe flexibilidad en el proyecto, y los beneficios de la misma se manifiestan en la presencia de irreversibilidad e incertidumbre.

A continuación, se calculará el valor de la opción social de diferir el tramo $1 \mathrm{~B}$.

\section{OPCIÓN SOCIALDE DIFERIREL TRAMO $1 B$}

En el tramo $1 \mathrm{~B}$ vemos que aunque tenemos un VPN Social de inicio negativo (Cuadro No 03), de acuerdo a la TRI existe la flexibilidad de poder diferir la inversión 4 años, siendo entonces, este el tiempo de duración de la opción.

El modelo de valuación que se utilizará será el modelo binomial ya que tiene algunas ventajas sobre otros modelos. Los parámetros más importantes a determinar son la volatilidad y la tasa de interés libre de riesgo. Para el caso de la volatilidad, hemos visto que es posible que exista incertidumbre asociada a las estimaciones de los beneficios netos y por tanto, en el valor presente que mide el valor de estos beneficios. De esta manera, para estimar la desviación estándar $(\sigma)$ en el valor presente de los beneficios netos del proyecto del libramiento carretero, se supondrá que esta será similar a la desviación estándar (volatilidad histórica) del Producto Interno Bruto (PIB) nacional. De acuerdo al CEPEP, este supuesto se apoya en que para la proyección de los TDPA se aplica la tasa de crecimiento del PIB, considerando que en la mayoría de los casos, esta tasa mantiene una relación aproximada con la tasa de crecimiento de los flujos vehiculares. Para el libramiento carretero, conforme a la Secretaría de Comunicaciones y Transportes (SCT) de México, se estimó que el flujo vehicular (demanda potencial) crecería a una tasa del $3 \%$ anual.

Existe una regla con cierta validez que indica que se puede utilizar un período semejante al del vencimiento de las opciones que se están analizando. De esta manera, para el ejemplo del libramiento carretero se estimará la desviación estándar del PIB en el intervalo de tiempo 1993-1996, que corresponde al período de 4 años que se tiene para diferir la inversión en el tramo $1 \mathrm{~B}$.

La desviación estándar trimestral en el período 1993-1996 se calculó como otrimestral $=5.63 \%$, que en términos anuales se tiene $\sigma$ anual $=\sigma$ trimestral $\times$ $=11.26 \%$.

Por otra parte, en el caso de la tasa libre de riesgo se utilizaron los datos históricos de los Cetes a 28 días, por ser la tasa libre de riesgo más representativa del mercado financiero mexicano. Para encontrar el valor de esta tasa se calculó la media geométrica de los datos históricos de los rendimientos anualizados de los Cetes a 28 días en el período 1993-1996, que es igual al tiempo de vida de la opción social de diferir el tramo 1B. Ahora, puesto que la tasa libre de riesgo debe ser una tasa real, también se calculó la media geométrica de los datos históricos de la inflación anual en México en el mismo período.

La media geométrica de la serie de los Cetes está dada por $23.35 \%$, y la correspondiente a la serie de datos de la inflación tiene un valor de $16.46 \%$. Entonces, utilizando la siguiente expresión:

$$
\text { Tasa de Interés Real }=\left(\frac{1+\text { Tasa de Interés Nominal }}{1+\text { Tasa de Inflación }}\right)-1,
$$

Se encuentra que la tasa libre de riesgo real es de $5.92 \%$ anual, que en su forma continua es igual a: e $0.0592-1=0.06099$ ó $6.10 \%$ anual aprox.

Cabe decir, que estos dos parámetros, la volatilidad y la tasa libre de riesgo, se supondrán constantes durante la vida de la opción. De esta manera, cuando se tienen horizontes de tiempo lejanos en un modelo 
más sofisticado se debe modelar estos parámetros como variables aleatorias.

Así, se tienen los siguientes parámetros para valuar la opción social de diferir el tramo 1B:

Monto (Inversión) Social $=\$ 34,685,924$

Valor Presente de los Beneficios Netos $=\$ 34,341,718$

VPN Social $=-\$ 34,685,924+\$ 34,341,718=-\$ 344,206$

$\mathrm{S} 0=\$ 34,341,718$

$\sigma=11.26 \%$

$\mathrm{r}=6.10 \%$

$\mathrm{T}=4$ años

$\delta \mathrm{t}=\mathrm{T} / \mathrm{N}=$ Vencimiento en años/Número de perío$\operatorname{dos}=4 / 4=1$

Para valuar esta opción mediante el método binomial, utilizamos el siguiente conjunto de expresiones para los parámetros $\mathrm{u}, \mathrm{d}$ y q (Wilmott et al., 1999):

$$
\left\{\begin{array}{l}
u=e^{r \delta t}\left(1+\sqrt{e^{\sigma^{2} \delta t}-1}\right) \\
d=e^{r \delta t}\left(1-\sqrt{e^{\sigma^{2} \delta t}-1}\right) \\
q=\frac{1}{2} .
\end{array}\right.
$$

Al sustituir los datos correspondientes obtenemos que:

$$
\mathrm{u}=1.1830, \mathrm{~d}=0.9428 \text { y q }=1 / 2
$$

De esta manera, podemos plantear el árbol binomial del activo subyacente (Valor Presente de los Bene- ficios Netos) como lo muestra la Figura No 1. Por ejemplo, a partir de los datos tenemos que $\mathrm{S} 0$ es igual a $\$ 34,341,718$ y que, al transcurrir un año, puede ascender $\mathrm{S}_{0} \mathrm{u}=\$ 34,341,718 \times 1.1830=\$ 40,626,252$ o, en cambio, descender a $\mathrm{S}_{0} \mathrm{~d}=\$ 34,341,718 \times 0.9428$ $=\$ 32,377,372$. Al transcurrir dos años, vemos que $S_{0}$ puede tener tres valores que son: $\mathrm{S}_{0} \mathrm{u}^{2}=\$ 34,341,718$ $\times 1.1830^{2}=\$ 48,060,857$, S0ud $=\$ 34,341,718 \times$ $1.1830 \times 0,9428=\$ 38,302,431$ y S0d ${ }^{2}=\$ 34,341,718$ $\times 0.9428^{2}=\$ 30,525,386$.

Figura No 1: Árbol Binomial del Activo concerniente al Tramo 1B

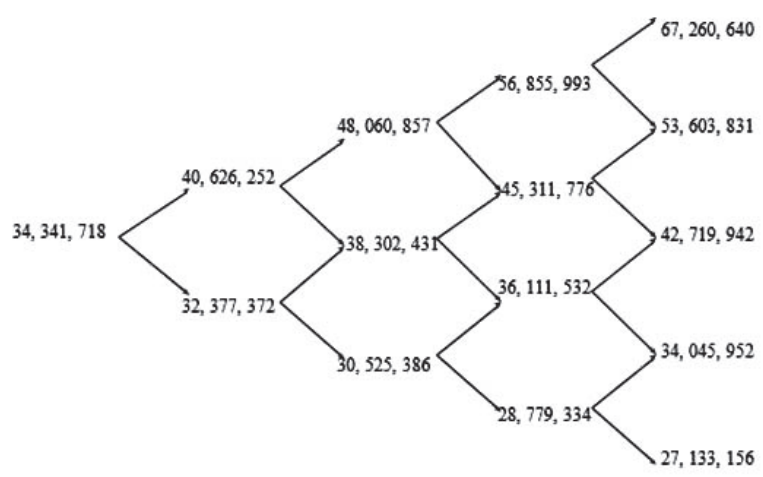

Fuente: Elaboración propia.

Sin embargo, el cálculo de estos valores esperados está suponiendo que se van a obtener los Beneficios Netos de los años 1998, 1999, 2000 y 2001 de la operación del tramo 1B (ver Cuadro No 04), lo que es falso puesto que al retrasar la inversión 4 años se renuncia a estos beneficios. Por lo tanto, a estos valores esperados se les debe sustraer el costo del retraso. 
Cuadro $\mathrm{N}^{\circ}$ 4: Momento Óptimo de Operación para el Tramo 1B

\begin{tabular}{|c|c|c|c|c|}
\hline Año & $\begin{array}{c}\text { Beneficio Neto } \\
\text { (\$) }\end{array}$ & $\begin{array}{l}\text { Anulidad de la } \\
\text { Inversión (Ir) }\end{array}$ & $\begin{array}{c}\text { Tasa de Rentabilidad } \\
\text { Inmediata }\end{array}$ & $\begin{array}{c}\text { Tasa Social de } \\
\text { Descuento }\end{array}$ \\
\hline $\begin{array}{l}1998 \\
1999 \\
2000\end{array}$ & $\begin{array}{l}4,946,607 \\
5,095,005 \\
5,247,855\end{array}$ & $\begin{array}{l}6,243,466 \\
6,243,466 \\
6,243,466\end{array}$ & $\begin{array}{l}14,26 \% \\
14,69 \% \\
15,13 \% \\
\end{array}$ & $\begin{array}{l}18 \% \\
18 \% \\
18 \% \\
\end{array}$ \\
\hline $\begin{array}{l}2001 \\
\mathbf{2 0 0 2} \\
2003 \\
2004 \\
2005 \\
\end{array}$ & $\begin{array}{c}5,405,291 \\
\mathbf{5 , 5 6 7 , 4 5 0} \\
5,734,473 \\
5,906,507 \\
6,083,702 \\
\end{array}$ & $\begin{array}{c}5,549,748 \\
\mathbf{5 , 5 4 9 , 7 4 8} \\
5,549,748 \\
5,549,748 \\
5,549,748 \\
\end{array}$ & $\begin{array}{c}15,58 \% \\
\mathbf{1 6 , 0 5} \% \\
16,53 \% \\
17,03 \% \\
17,54 \% \\
\end{array}$ & $\begin{array}{l}16 \% \\
16 \% \\
16 \% \\
16 \% \\
16 \% \\
\end{array}$ \\
\hline $\begin{array}{l}2006 \\
2007 \\
2008 \\
2009 \\
2010 \\
\end{array}$ & $\begin{array}{l}6,266,214 \\
6,454,200 \\
6,647,826 \\
6,847,261 \\
7,052,679 \\
\end{array}$ & $\begin{array}{l}4,856,029 \\
4,856,029 \\
4,856,029 \\
4,856,029 \\
4,856,029 \\
\end{array}$ & $\begin{array}{l}18,07 \% \\
18,61 \% \\
19,17 \% \\
19,74 \% \\
20,33 \% \\
\end{array}$ & $\begin{array}{l}14 \% \\
14 \% \\
14 \% \\
14 \% \\
14 \% \\
\end{array}$ \\
\hline $\begin{array}{l}2011 \\
2012 \\
2013 \\
2014 \\
2015 \\
2016 \\
2017\end{array}$ & $\begin{array}{l}7,264,259 \\
7,482,187 \\
7,706,652 \\
7,937,852 \\
8,175,987 \\
8,421,267 \\
8,673,905\end{array}$ & $\begin{array}{l}4,162,311 \\
4,162,311 \\
4,162,311 \\
4,162,311 \\
4,162,311 \\
4,162,311 \\
4,162,311\end{array}$ & $\begin{array}{l}20,94 \% \\
21,57 \% \\
22,22 \% \\
22,88 \% \\
23,57 \% \\
24,28 \% \\
25,01 \%\end{array}$ & $\begin{array}{l}12 \% \\
12 \% \\
12 \% \\
12 \% \\
12 \% \\
12 \% \\
12 \%\end{array}$ \\
\hline
\end{tabular}

Fuente: Elaboración propia.

Por ejemplo, para encontrar el costo del retraso en el primer período vemos que el valor presente de $\$ 4,946,607$ que se reciben en el primer año de operación del tramo $1 \mathrm{~B}$, descontados a la tasa social de descuento (costo de oportunidad de los recursos públicos) del $18 \%$ es igual a $\$ 4,192,040$. Por tanto, el costo del retraso es igual a $\$ 4,192,040 / \$ 34,341,718$ $=12.21 \%$ del valor de $\$ 40,626,252$ y $\$ 32,377,372$; es decir, $\$ 4,960,465$ y $\$ 3,953,277$, respectivamente.

De la misma forma, para encontrar el costo del retraso en el segundo período vemos que el valor presente de $\$ 5,095,005$ que se reciben en el segundo año, descontados a la tasa social de descuento del $18 \%$ es igual a $\$ 3,659,153$. Entonces, el costo del retraso es igual a $\$ 3,659,153 / \$ 34,341,718=10.66 \%$ del valor de $\$ 48,060,857, \$ 38,302,431$ y $\$ 30,525,386$, es decir, $\$ 5,123,287, \$ 4,083,039$ y $\$ 3,254,006$, respectivamente.

Así pues, en la Figura № 2 se muestra el árbol binomial correspondiente donde se considera el costo del retraso. Por ejemplo, en el primer período se tienen los valores $\$ 40,626,252-\$ 4,960,465$ $=\$ 35,665,787$ y $\$ 32,377,372-\$ 3,953,277=$ $\$ 28,424,095$.

Por otro lado, el monto social $\$ 34,685,924$ para realizar el tramo $1 \mathrm{~B}$ crecerá cada año, al menos, a una tasa igual a la libre de riesgo. Así, para los años 1, 2, 3 y 4 el monto social será $\$ 36,801,765, \$ 39,046,673$, $\$ 41,428,520$ y $\$ 43,955,660$, respectivamente.

La mayoría de las opciones de diferir un proyecto son americanas. Por consiguiente, la decisión sobre si ejercer o no la opción deberá postergarse hasta que el valor por tiempo de esta sea nulo.

Los pagos finales de la opción social de diferir el tramo $1 \mathrm{~B}$ son los siguientes:

$$
\begin{aligned}
& \text { máx }\{61,704,911-43,955,660,0\}=\$ 17,749,251 \\
& \text { máx }\{49,176,154-43,955,660,0\}=\$ 5,220,494 \\
& \text { máx }\{39,191,275-43,955,660,0\}=\$ 0 \\
& \text { máx }\{31,233,756-43,955,660,0\}=\$ 0 \\
& \operatorname{máx}\{24,891,957-43,955,660,0\}=\$ 0
\end{aligned}
$$


Figura No 2: Árbol Binomial del Activo menos el Costo del Retraso concerniente al Tramo 1B

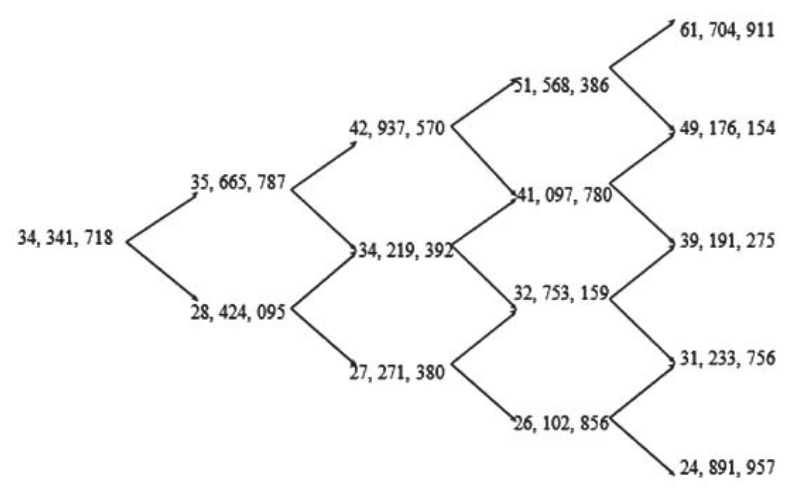

Fuente: Elaboración propia.

Luego, si se retrocede en el árbol binomial utilizando la fórmula recursiva (Wilmott et al., 1999):

$V_{j}^{n-1}=\operatorname{máx}\left\{e^{-r \delta \delta}\left[q V_{j+1}^{n}+(1-q) V_{j}^{n}\right] f_{j}^{n-1}\right\} \quad 0 \leq j \leq n-1$,

Obtenemos el árbol de la Figura No 3.

Por consiguiente, el VPN Social total con la opción social de diferir 4 años el tramo 1 B es igual a:

VPN Social total $=\$ 1,891,693$

De este modo, para calcular el valor de la opción social de diferir se toma la diferencia entre el VPN Social con la opción de diferirlo $(\$ 1,891,693)$ y el VPN Social estático que no considera la demora $(-\$ 344,206)$, es decir, Opción Social de Diferir 4 Años el Tramo

$$
1 \mathrm{~B}=\$ 1,891,693-(-\$ 344,206)=\$ 2,235,899
$$

Figura No 3: Árbol Binomial para la Opción Social de Diferir el Tramo 1B

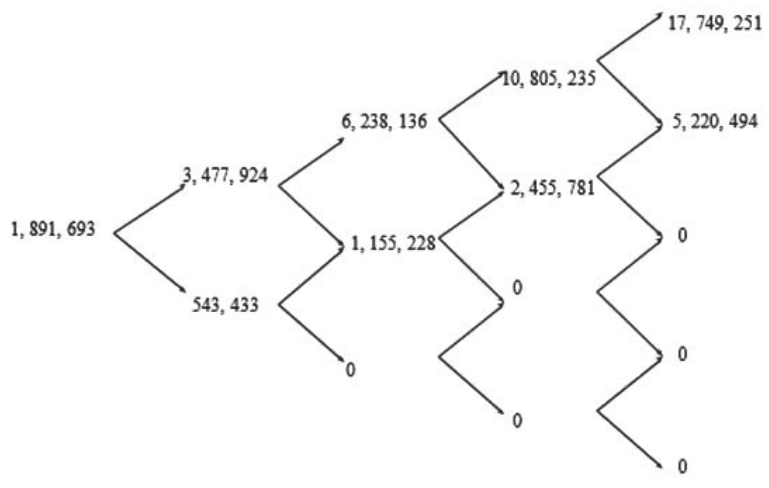

Fuente: Elaboración propia.
En otras palabras, el VPN Social total es igual al VPN Social estático más una componente dinámica dada por el valor de la opción, es decir,

$$
\$ 1,891,693=-\$ 344,206+\$ 2,235,899 \text {. }
$$

Como ya se mencionó, los beneficios de un proyecto de infraestructura vial están en función únicamente del tiempo y no dependen del momento en el que inicie el mismo. De hecho, desde esta perspectiva de la evaluación social de proyectos, el cálculo del VPN Social no es suficiente para determinar la rentabilidad de un proyecto de esta naturaleza y, por lo tanto, se utiliza el criterio de la TRI para definir el momento óptimo de la inversión. Sin embargo, como ya vimos, la metodología de opciones reales nos permite conocer el verdadero valor de un proyecto de infraestructura vial cuando este es propuesto por alguna dependencia del sector público, considerando la flexibilidad de que se puede diferir su ejecución un cierto tiempo (debido al régimen de exclusividad para invertir del sector público y definido por la TRI), y cuando existe irreversibilidad e incertidumbre.

Para que la riqueza de la sociedad aumente al máximo cuando se pretende llevar a cabo un proyecto de infraestructura vial, la evaluación social debe considerar el valor de la opción social de diferir cuando la misma exista. Por lo tanto, en la evaluación social de un proyecto de infraestructura vial se pueden emplear la TRI y el concepto del Valor Presente Neto Extendido Social para determinar la rentabilidad de un proyecto de este tipo.

Por último, cabe decir que en el ejemplo del libramiento carretero sólo se determinó el valor de la opción social de diferir el tramo 1B. Sin embargo, también se puede valuar esta opción cuando existe un VPN Social cercano a cero y positivo y la incertidumbre en los flujos del proyecto es alta, ya que se puede ganar más al tener la flexibilidad de poder diferirlo y al tener derechos exclusivos, por las mismas razones que los inversionistas no siempre ejercen la opción porque está in the money. 


\section{CONCLUSIONES}

1. En lo que respecta a la evaluación social de proyectos, en México no existen los precios sociales, con excepción de la tasa social de descuento, la cual ha sido motivo de controversia al ser utilizada como tasa de descuento en el análisis costo-beneficio, debido a las distintas concepciones acerca de las modalidades de funcionamiento del sector público y a diversos argumentos teóricos y prácticos.

2. En el mismo sentido, en la evaluación social no se tiene una cultura de la incorporación del riesgo en la planeación de los proyectos.

3. Parala modelación de la opción social de diferir un proyecto se tuvieron que hacer algunos supuestos, como el hecho de que la volatilidad y que la tasa de interés libre de riesgo eran constantes durante el tiempo de vida de la opción. En realidad, la economía mexicana aún es muy vulnerable a factores macroeconómicos internos y externos, viéndose por tanto comprometidos estos parámetros clave en la valuación de una opción.

4. En el cálculo de la volatilidad se hizo una importante suposición, al establecer que en el proyecto de infraestructura vial la misma correspondía a la volatilidad histórica del Producto Interno Bruto (PIB), este supuesto se apoya en que para la proyección de la demanda y por tanto, de los beneficios netos futuros, se aplica la tasa de crecimiento del PIB. No obstante, este supuesto puede darle debilidad a la estructura del modelo, pues podría no reflejar una variabilidad fiel de los beneficios netos futuros del proyecto.

5. Así pues, identificar, formular evaluar, y llevar a cabo los proyectos de inversión pública más rentables representa sin duda una de las mejores opciones para el desarrollo de cualquier país. De esta manera, emplear la metodología de opciones reales puede ayudar a garantizar que se obtenga la máxima rentabilidad en los proyectos de inversión pública.

\section{REFERENCIAS BIBLIOGRÁFICAS}

1. AMRAM, M. y KULATILAKA, N., Real Options. Managing Strategic Investment in an Uncertain World, (Harvard Business School Press, Boston, Massachusetts).

2. CEPEP (2004) "Apuntes Sobre Evaluación Social de Proyectos”, Banco Nacional de Obras y Servicios Públicos, S.N.C. (BANOBRAS), Centro de Estudios para la Preparación y Evaluación Socioeconómica de Proyectos (CEPEP), México.

3. GALA P., J. "La Evaluación Social de Proyectos. ¿Qué es?, ¿Para qué sirve?” (Documento de Trabajo, Centro de Estudios para la Preparación y Evaluación Socioeconómica de Proyectos (CEPEP), México).

4. FONTAINE, E.R. (2005) "Evaluación Social de Proyectos” Alfaomega - Ediciones Universidad Católica de Chile, México.

5. ORTIZ, E., Finanzas y Productos Derivados: Contratos adelantados, Futuros, Opciones y Swaps (mimeo, UNAM).

6. SCHWARTZ, E. S. y TRIGEORGIS, L. (2001) Real Options and Investment under Certainty: Classical Reading and Recent Contributions. The MIT Press.

7. ZHAO, T., SUNDARARAJAN, S.K. y TSENG, C. (2004) "Highway Development Decision-Making under Uncertainty: A Real Option Approach", Journal of Infrastructure Systems (pp. 23-32)

8. WILMOTT, P., DEWYNNE, J. y HOWISON, S. (1999) The Mathematics of Financial Derivatives. A Student Introduction. Cambridge University Press. 